\section{Controlled study of correlation of biomechanical profile of hemiparetic patients with distance travelled in six minutes}

\author{
Laís Moreira Moura, 1 \\ Mônica Maria Pena Quintão,1,2 \\ Karen Santos R. de Carvalho,1 \\ Beatriz Cantanhede Carrapatoso, 1 \\ Sabrina Lindenberg L. Malfacini, ${ }^{1}$ \\ André Custódio da Silva, 1 \\ Marco Orsini,3,4 \\ Osvaldo J.M. Nascimento, 3 \\ Sergio S.M.C. Chermont1,2 \\ 1The Heart Failure Clinic, School Clinic of \\ Physiotherapy, Serra dos Órgãos \\ University Center, Teresópolis; \\ 2Post Graduate Program in \\ Cardiovascular Sciences, Fluminense \\ Federal University, Niterói; \\ 3Fluminense Federal University, Niterói; \\ 4Masters Program in Science \\ Rehabilitation, Centro Universitário \\ Augusto Motta, Bonsucesso, Rio de \\ Janeiro, Brazil
}

\section{Abstract}

The six-minute walking test (6MWT) is used to assess exercise tolerance that is associated with motor function of the lower limbs in hemiparetic patients. It is suggested that, for post-stroke subjects, performance in the 6MWT may be limited by biomechanical and cardiovascular factors. Our aim is to determine the correlation between the six-minute walk distance (6MWD) and the biomechanical profile of hemiparetic patients. During this crosssectional controlled study, 10 hemiparetic patients with heart failure underwent 6MWT (ATS protocol). Tonus (Ashworth Scale) and goniometry of the lower limbs were measured. The average of $6 \mathrm{MWD}$ in two tests was $279 \pm 8$ $m$. There was a negative correlation between the degree of spasticity for both the sural triceps $(\mathrm{r}=-0.57, \mathrm{P}<0.05)$, quadriceps $(\mathrm{r}=-0.58$, $\mathrm{P}<0.05)$ and the limitation in ankle dorsiflexion and the 6MWD $(\mathrm{r}=-0.76, \mathrm{P}<0.05)$. Also, there was correlation between hip extension and ankle dorsiflexion limitations with 6MWD $(\mathrm{r}=0.66, \mathrm{P}<0.05),(\mathrm{r}=0.77, \mathrm{P}<0.05)$. The negative correlation between the highest spasticity in paretic limb and the 6MWD and the correlation between the lower movement range of paretic hip and ankle suggest association with these factors and gait velocity in 6MWT. Loss percentage represents the percentage calculation between distance traveled and the dis- tance predicted achieved by patients. In this study, the negative correlation between the percentage of loss of 6MWD and the limitation in the ankle dorsiflexion movement suggests that for a minor motion arch of the ankle, there is a higher percentage of walking distance loss foretold.

\section{Introduction}

Stroke (cerebrovascular accident, CVA) can be defined as a condition where cerebral blood flow reduction occurs leading to decreased availability of glucose and oxygen to the brain cells, causing cell death and spread of the brain injury. It is considered the third most common cause of death after cardiovascular disease and cancer in developed countries. ${ }^{1}$

Among the main changes caused by the sequelae of stroke, are the changes in muscle tone, depletion of postural control mechanisms, besides inadequate synergies movements, factors that determine patterns of unsuitable ambulation. ${ }^{2}$

The gait of a hemiparetic individual has been described as slow and asymmetric. The decrease in walking speed can be linked to a number of factors such as: limitation of cadence, step length and gait cycle, decreased range of motion and stride length, inability to perform selective movements in the joints of the lower limbs and a reduction in the swing phase. The asymmetry is due to the time difference in support between the lower limbs, which can lead to an energy consumption resulting in decreased tolerance to exercise. 3,4

Most patients with stroke conditions showed spasticity, which often interferes significantly in achieving goals established by the rehabilitation programs. The Modified Ashworth Scale is widely used in the evaluation of spasticity. Its acceptance is due to its reliability and reproducibility. 5 The six-minute walk test (6MWT) is a submaximal exercise test, wherein the distance walked in six minutes (6MWD) during a 6MWT used to assess tolerance and exercise capacity in several chronic diseases, because the ability to walk is closely linked to the activities performed in everyday life. The 6MWT evaluates integrated responses of all physiological systems involved in the exercise that include cardiovascular and respiratory systems, motor units and peripheral skeletal muscles. ${ }^{6}$ The $6 \mathrm{MWT}$ performance may be limited by both biomechanical and neuromuscular factors, as the cardiovascular response to this type of exercise. ${ }^{7,8}$ Therefore, the 6MWT can be associated with gait speed, balance and motor function of the lower limbs in patients with sequelae of stroke. However little is known about the response of this submaximal test in these patients.
Correspondence: Sergio Chermont, School Clinic of Physiotherapy, University Course of Physiotherapy - UNIFESO, Av. Delfim Moreira, 2211 Vale Paraíso, Teresópolis, RJ 25953-184, Brazil.

Tel.: +55.21 .2152 .4900$

E-mail: schermont@yahoo.com.br

Key words: Stroke; six-minute walk test; sixminute walk distance; biomechanical profile.

Contributions: the authors contributed equally.

Conflict of interest: the authors declare no potential conflict of interest.

Received for publication: 23 July 2014.

Revision received: 18 March 2015.

Accepted for publication: 15 June 2015

This work is licensed under a Creative Commons Attribution NonCommercial 3.0 License (CC BYNC 3.0).

(C) Copyright L.M. Moura et al., 2015

Licensee PAGEPress, Italy

Neurology International 2015; 7:5417

doi:10.4081/ni.2015.5417

\section{Materials and Methods}

The protocol of this present study was approved by the ethics and research committee at Serra dos Orgãos University Center. All participants of this project received detailed information about the purpose of the study and signed a free and informed consent form according to resolution 466/12.

Statistical analysis was performed through the Student ANOVA one way, student-t test and Pearson correlation coefficient. The sample size calculation was made based on previous publications that showed this type of intervention. For purposes of this magnitude and to secure the statistical power of 0.8 and an alpha error of 0.05 , the minimum sample size was 13 individuals. The $P$ value considered significant was $\leq 0.05$. The study design followed a crosssectional, controlled protocol. The sample consisted of 17 hemiparetic patients diagnosed with CVA, under treatment at the School of Physiotherapy Clinic at Serra dos Órgãos University Center, Brazil. Out of 17 patients only 10 were able to complete the proposed protocol. Patients were selected according to inclusion and exclusion criteria. The patients were evaluated through physical examination and submitted to the 6MWT, following the protocol of the American Association of Cardiovascular and Pulmonary Rehabilitation (AACVPR). On physical examination the tone of the muscles was evaluated in the passive 
mode. Placing the patient in a supine position. To test a muscle that flexes a joint mainly, the set was placed in a maximum flexed position and move to a position of maximum extension over a second (count one thousand). All movements were repeated three times, and as a reference for measuring the tone, the Modified Ashworth Scale was used.

I. The gluteus minimus was evaluated with the patient lying in the supine position, lower limbs (LL) extended, performing passive abduction and medial rotation of the segment

II. The gluteus maximus with the patient lying in the lateral position, lower limbs extended, passive extension of the thigh was performed.

III. The femoral quadriceps with the patient lying in the supine position lower limbs extended, passive flexion/extension in the follow-up was performed, and sural triceps, with the patient lying in the supine position extended lower limbs, passive dosiflexion/plantarflexion was performed.

The range of motion of the lower limbs was also evaluated by goniometry with a goniometer where were measured: hip flexion, hip extension, knee flexion, knee extension, plantar flexion and dorsiflexion.

The 6MWT was performed by marking the distance traveled and the number of turns for six minutes using the physical space of a leveled corridor of 30 meters in length, continuously monitoring signals of dyspnea, fatigue, vertigo/dizziness, chest pain or lower limbs, and every two minutes, heart rate (HR), oxygen saturation (Sp02) and feeling of perceived

Table 1. Characteristics of patients.

\begin{tabular}{lc} 
Characteristic & $\mathrm{N}$. \\
Men/Women & $7 / 3$ \\
Age & $50 \pm 18$ \\
\hline Hemiparesis right/left & $4 / 6$ \\
Injury time (months) & $28 \pm 18$ \\
\hline Six-minute walk distance (average) & $279 \pm 8$ \\
\hline
\end{tabular}

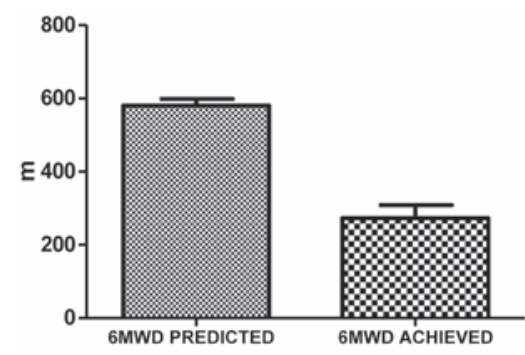

Figure 1. Difference between the walking distance predicted for patients and the distance achieved $(P=0.0001)$. exertion (Borg Scale modified), and systolic blood pressure (SBP) and diastolic (DBP), respiratory rate (RR), auscultation lung (AP), cardiovascular auscultation (ACV), distance walked in six minutes (6MWD) and the other variables provided by the Protocol of AACVPR.

\section{Results}

Patient characteristics are shown in Table 1. Data were expressed as mean \pm standard deviation.

The average $6 \mathrm{MD}$ of the two tests was $279 \pm 8$ $\mathrm{m}$. There was a significant difference between the walking distance predicted for these patients and the distance achieved (Figure 1 and Table 2).

In Figure 2 the main results of the associa- tion between 6MWD and biomechanical characteristics of patients are related.

There was a negative correlation between greater spasticity and 6MWD both for the sural triceps $(\mathrm{r}=-0.57, \mathrm{P}<0.05)$ and for the quadriceps $(\mathrm{r}=-0.58, \mathrm{P}<0.05)$. Also, there was correlation between the limitation in hip extension movement and 6MWD $(\mathrm{r}=0.66, \mathrm{P}<0.05)$, besides correlation between the limitation in dorsiflexion movement of the ankle and 6MWD $(\mathrm{r}=0.77, \mathrm{P}<0.05)$. Figure 2 shows the interference of the biomechanical characteristics of the patients evidenced by increased spasticity and decreased range of motion in the distance traveled.

In the present study the 6MWD achieved by patients was far below the one predicted $581 \pm 59$ causing a percentage of loss in $6 \mathrm{MWD}$ of $53 \pm 18 \%$. It was observed that patients who had a higher percentage of loss in 6MWD had

Table 2. Evaluation of tonus.

\begin{tabular}{lcccc}
\hline Patient & Minimum gluteus & Maximus gluteus & Quadriceps & Triceps surae \\
1 & 1 & 2 & 2 & 2 \\
2 & 2 & 0 & 0 & 2 \\
\hline 3 & 3 & 1 & 1 & 3 \\
4 & 2 & 1 & 1 & 3 \\
\hline 5 & 2 & 1 & 1 & 3 \\
6 & 1 & 1 & 1 & 2 \\
\hline 7 & 2 & 2 & 0 & 3 \\
8 & 3 & 3 & 3 & 3 \\
\hline 9 & 2 & 2 & 1 & 3 \\
10 & 2 & 2 & 3 & 4 \\
\hline
\end{tabular}
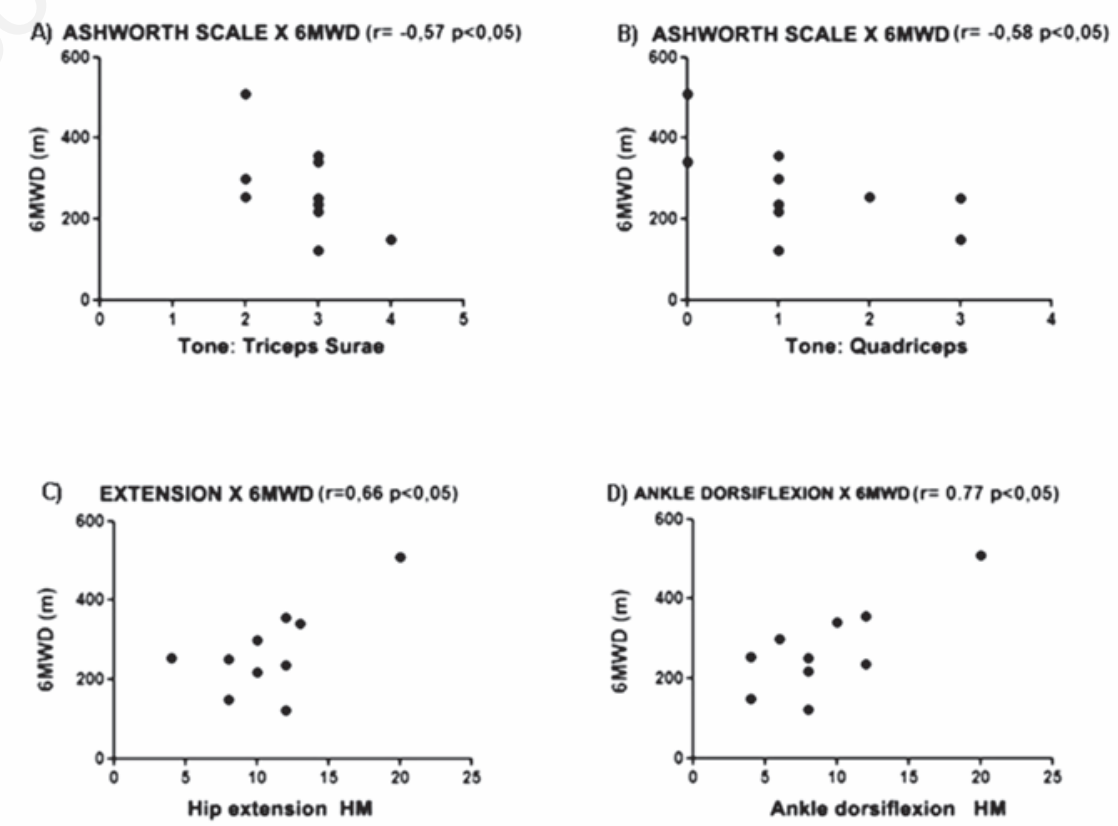

Figure 2. Results of the association between six-minute walk distance and biomechanical characteristics of patients. 
lesser degree of dorsiflexion range of motion, generating a negative correlation between the percentage of loss of 6MWD and the limitation in the ankle dorsiflexion movement $(\mathrm{r}=-0.76$, $\mathrm{P}<0.05$; Figure 3 ).

\section{Discussion}

The percentage decrease in 6MWD of $53 \pm 18 \%$ showed a significant involvement of functional capacity in these patients. In the study by Eng et al. ${ }^{9}$ an association between higher degrees of spasticity and less distance walked was found, reflecting a pattern of walking at a slower speed. In the present study a lower 6MWD was found with cutoff far below the 6 MWD predicted of $581 \pm 59$.

The Ashworth scale used in the lower limbs to assess hemiparesis spastic component was cited in the review Pandyan et al.10 and used in the study by Eng et al. ${ }^{9}$ where showed values around 1, different from the present study that as a result denoted levels around 2 for sural triceps and for lquadríceps demonstrating that this present study bothered to discern the origin of this factor interference through the different levels of spasticity according to each muscle group involved and its respective joint participant in the gait. Therefore, there was a negative correlation between greater spasticity and the 6MWD both for the Sural Triceps $(\mathrm{r}=-0.57, \quad \mathrm{P}<0.05)$ as for the Quadriceps $(\mathrm{r}=-0.58, \mathrm{P}<0.05)$.

The study by Corrêa et al.11 measured the electromyographic pattern of post-stroke

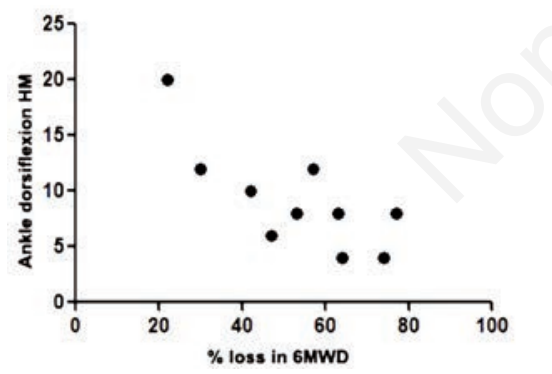

Figure 3. Correlation between the percentage of loss of six-minute walk distance and the limitation in the ankle dorsiflexion movement. patients. In this study a reduction in the activity of the affected ankle muscles was reported. However, in spite of the electrogoniometry had also been taken, the results were not presented, which differs from the present study that showed significant decrease in range of motion for dorsiflexion with a significant correlation with $6 \mathrm{MWD}$.

A negative correlation that was observed in the present study between 6MWD and range of motion of dorsiflexion, has determined that for a smaller range of motion of the ankle there is a greater percentage loss of distance traveled predicted for a normal value in the walk test.

\section{Conclusions}

We observed in this study that patients with stroke have decreased aerobic capacity that results in physical deconditioning, affecting the quality of life of these patients.

The 6MWT is essential for the evaluation of exercise tolerance in several pathologies importance. In addition to reflecting exercise tolerance, the 6MWT is considered safe, cheap and best reflects the activities of daily life for most patients. The 6MWD obtained in the $6 \mathrm{MWT}$ is considered a strong prognostic variable value for these patients.

The behavior of the 6MWT in hemiparetic patients was based on the following pattern: a cutoff of $280 \mathrm{~m}$ to $6 \mathrm{MWD}$, to determine exercise tolerance in this specific population.

There was a correlation between the 6MWD and the limitation of the ankle movement and hip extension, and between the 6MWD and the triceps surae tone and quadriceps thus reflecting interference of the biomechanical pattern in 6MWD.

The 6MWT, clearly, can be used to determine the exercise intolerance in some patients. This study demonstrated that the use of the 6MWT in hemiparesis is able to determine the acute effects of submaximal exercise on cardiopulmonary function and biomechanical profile of hemiparetic patients and it can be used in functional diagnosis of these patients to determine exercise tolerance, guided treatment and to measure the progress of this patient during rehabilitation.

Future investigations and studies can be used to explore the potential clinical impact of this original study.

\section{References}

1. Kaur H, Prakash A, Medhi B. Drug therapy in stroke: from preclinical to clinical studies. Pharmacology 2013;92:324-34.

2. Meneghetti CHZ, Delgado GM, Pinto FD, et al. Equilíbrio em indivíduos com Acidente Vascular Encefálico: Clínica Escola de Fisioterapia da Uniararas. Rev Neuroc 2009;17:14-8

3. Von Schroede, HP, Coutts RD, Lyden PD, et al. Gait parameters following stroke: a practical assessment. J Rehabil Res Dev 1995;32:25-31.

4. Yang Y, Yen JG, Wang RY, et al. Gait outcomes after additional backward walking training in patients with stroke: a randomized controlled trial. Clin Rehabil 2005;19: 264-73.

5. Lima FPS, Lima M0, de Freitas TT, et al. Revisão da literatura: espasticidade. 2004. Available from: http://www.inicepg.univap.br/cd/INIC_2004/trabalhos/epg/pdf/EP G4-24R.pdf

6. Resqueti RV, Oliveira GWS, Dourado Jr ME, et al. Confiabilidade do teste da caminhada de seis minutos em pacientes com miastenia gravis generalizada. Rev Fisioter Pesquis 2009;16:223-8.

7. Pohl PS, Duncan PW, Perera S, et al. Influence of stroke-related impairments on performance in 6-minute walk test. $\mathrm{J}$ Rehabil Res Dev 2002;39:439-44.

8. Fulk GD, Echternach JL, Nof L, O'Sullivan S. Clinometric properties of the sixminute walk test in individuals undergoing rehabilitation poststroke. Physiother Theory Pract 2008;24:195-204.

9. Eng JJ, Chu KS, Dawson AS, et al Functional walk tests in individuals with stroke: relation to perceived exertion and myocardial exertion. Stroke 2002;33:75661 .

10. Pandyan AD, Johnson GR, Price CI, et al. A review of the properties and limitations of the Ashworth and modified Ashworth Scales as measures of spasticity. Clin Rehabil 1999;13:373-83.

11. Corrêa FI, Soares F, Andrade DV, et al. [Muscle activity during gait following stroke]. Arq Neuropsiquiatr 2005;63:84751. [Article in Portuguese]. 